\title{
NDRG1 Targets TAF15 to Promote Endothelial Dysfunction and Hypoxia-Induced Pulmonary Hypertension
}

\section{Chengwei Li}

Huashan Hospital Fudan University

\section{Liang Dong}

Huashan Hospital Fudan University

Ning Zhu

Huashan Hospital Fudan University

\section{Xiujuan Zhang}

Huashan Hospital Fudan University

\section{Ruzetuoheti Yiminniyaze}

Huashan Hospital Fudan University

\section{Gulinuer Wumaier}

Huashan Hospital Fudan University

\section{Xiaomin Wei}

Huashan Hospital Fudan University

Jing Li

Huashan Hospital Fudan University

Junzhu Lv

Huashan Hospital Fudan University

Jingwen Xia

Huashan Hospital Fudan University

\section{Shuanghui Li}

Huashan Hospital Fudan University

\section{Yuzhen Zeng}

Huashan Hospital Fudan University

\section{Jing Wang}

Huashan Hospital Fudan University

\section{Daibing Zhou}

Huashan Hospital Fudan University

Shengqing Li ( $\nabla$ shengqingli@hotmail.com )

Huashan Hospital Fudan University https://orcid.org/0000-0002-0570-2656 
Research

Keywords: N-myc downstream regulated gene-1, TATA-Box binding protein associated factor 15, hypoxiainduced pulmonary hypertension, endothelial dysfunction, vascular remodeling

Posted Date: August 19th, 2021

DOl: https://doi.org/10.21203/rs.3.rs-794105/v1

License: (c) (i) This work is licensed under a Creative Commons Attribution 4.0 International License.

Read Full License 


\section{Abstract}

Background: The mechanism underlying vascular remodeling of hypoxia-induced pulmonary hypertension $(\mathrm{HPH})$ is not fully elucidated. We hypothesized that hypoxia promotes expression of N-myc downstream regulated gene-1 (NDRG1) in human pulmonary arterial endothelial cells (HPAECs), which in turn leads to endothelial dysfunction and contributing to $\mathrm{HPH}$.

Methods: Lung samples were obtained from qualified patients and HPH rat models. Quantitative polymerase chain reactions, western blotting and immunohistochemistry were used to measure the expression of NDRG1. EdU incorporation assays, cell counting kit-8 (CCK-8) assays, transwell migration assays, and matrigel assays were conducted to detect the role of NDRG1 in HPACE function in vitro. HPH models were established in SD rats and were treated with plasmids expressing short hairpin RNAs (shRNAs) to silence NDRG1. The candidate binding partner(s) of NDRG1 was screened and validated via co-immunoprecipitation and immunofluorescence staining.

Results: NDRG1 is up-regulated by hypoxia in a time-dependent manner in HPAECs. Expression of NDRG1 was increased in lung tissues of HPH patient and rat model. In vitro, silencing NDRG1 attenuated proliferation, migration and tube formation of HPAECs under hypoxia, while NDRG1 over-expression promoted these behaviors of HPAECs in normoxia. NDRG1 knock-down alleviated vascular remodeling and right ventricular hypertrophy in rat models of HPH. NDRG1 can directly interact with TATA-box binding protein associated factor 15 (TAF15) and promote its nuclear localization. Bioinformatics study found that Notch1 signaling was downstream of TAF15 in endothelial cells. TAF15 can promote HPAECs dysfunction via binding to Notch1 promoter region and subsequently increasing Notch1 expression.

Conclusions: Taken together, hypoxia-induced up-regulation of NDRG1 contributes to endothelial dysfunction and HPH development through TAF15 upregulation of Notch1, suggesting the applicability of targeting NDRG1 in clinical treatment of $\mathrm{HPH}$.

\section{Introduction}

Pulmonary hypertension $(\mathrm{PH})$ is a pathophysiological syndrome characterized by an increase in mean pulmonary arterial pressure (mPAP) $\geq 25 \mathrm{mmHg}$ at rest, which can complicate the majority of primary diseases [1, 2]. Hypoxia-induced $\mathrm{PH}(\mathrm{HPH})$ is a high-incidence type of $\mathrm{PH}$, namely group $3 \mathrm{PH}$, and frequently occurs in patients with chronic lung diseases, such as chronic obstructive pulmonary disease (COPD), interstitial lung disease (ILD), and combined pulmonary fibrosis and emphysema (CPFE) [3]. HPH displays higher morbidity and mortality compared to other $\mathrm{PH}$ groups, and causes heavy economic burdens globally [4-6]. Long-term oxygen therapy can prolong survival but only partially meliorate pulmonary hypertension [7]. The use of approved drugs for group $1 \mathrm{PH}$ have not been shown to benefit $\mathrm{HPH}$ patients in randomized controlled trials [8]. Hence, a better understanding of disease mechanisms might be helpful to identifying new therapeutic targets for HPH. 
The characteristic pathophysiological changes in $\mathrm{HPH}$ are vasoconstriction and vascular remodeling induced by hypoxia [9]. The pathogenesis of vascular remodeling is complex, which necessarily includes the imbalance of vasoactive substances, abnormal energy metabolism of mitochondria, inflammation and immune response, gene susceptibility, epigenetics and other mechanisms [10]. In addition, each of the resident vascular cell types (ie, endothelial, smooth muscle, adventitial fibroblast) plays a specific role in the overall remodeling response [11-13]. In particular, the dysfunction of pulmonary artery endothelial cells (PAECs) in PH has been well-documented, with properties of aberrant proliferation, intimal endothelial cells migration and angiogenesis under hypoxia $[14,15]$.

To investigate the molecular mechanism of endothelial dysfunction during hypoxia, we compared pulmonary endothelial cells under normoxia and hypoxia condition, and identified that N-myc downstream regulated gene-1 (NDRG1) was remarkably upregulated in pulmonary endothelial cells when exposed to hypoxia. NDRG1 is expressed widely in human tissues, but is hardly detectable in normal endothelial cells [16]. NDRG1 plays multifunctional roles in cell growth, development, differentiation and stress responses $[17,18]$. However, the role of NDRG1 in the vascular remodeling of HPH remains largely elusive. In this study, we found that hypoxia promotes expression of NDRG1 in human pulmonary arterial endothelial cells (HPAECs), which in turn leads to endothelial dysfunction and contributes to the development of $\mathrm{HPH}$.

\section{Materials And Methods}

\section{Pulmonary surgical specimens}

The study was approved by the local ethical committee, and written informed consent was obtained from all patients to donate lung tissues. Surgical specimens of lung tissue were collected from patients with chronic lung disease-associated $\mathrm{PH}(\mathrm{n}=3)$, or corresponding non-tumor normal tissues without $\mathrm{PH}(\mathrm{n}=$ 3).

\section{Animal models and in vivo gene knockdown}

Adult male Sprague-Dawley rats weighing 150-200 g were purchased from the Shanghai SLAC Laboratory Animal Co. Ltd (Shanghai, China). All protocols and surgical procedures were approved by the Ethics Committee of Experimental Research at Fudan University Shanghai Medical College. Rat HPH model was established based on a previous study [19]. Briefly, Animals were divided randomly into the following two groups: 1) normoxia, and 2) chronic hypoxia. Rats in the normoxia group were housed at ambient barometric pressure for 28 days $\left(\sim 718 \mathrm{mmHg}, \mathrm{PO}_{2}\right.$ was $\left.\sim 150.6 \mathrm{mmHg}\right)$. Rats in the hypoxia groups were housed in a hypobaric hypoxia chamber depressurized to $380 \mathrm{mmHg}\left(\mathrm{PO}_{2}\right.$ was reduced to $79.6 \mathrm{mmHg}$ ) for $8 \mathrm{~h} /$ day for 28 days. All animals were housed under a 12:12 h light/dark cycle and free to sterile food and water. The room temperature was controlled at $22^{\circ} \mathrm{C} \pm 1^{\circ} \mathrm{C}$, and relative humidity was maintained at $50 \% \pm 5 \%$. 
Short hairpin RNA (shRNA) against rat NDRG1 and a negative control shRNA were designed and synthesized by GenScript, China. Entranster in vivo transfection reagent (Engreen Biosystem, China) was used as a vehicle for shRNA delivery according to the manufacturer's recommendations. The shRNAs against NDRG1 or negative control $(1 \mathrm{mg} / \mathrm{kg})$ were injected into the tail veins of SD rats per week during normoxia or hypoxia treatment.

\section{Echocardiography}

Echocardiography was performed under anesthesia (2\% isoflurane mixed with air), with the Vevo3100 Ultrasound system (GE Healthcare) and the $12 \mathrm{~S}$ rodent probe (GE Healthcare) to determine pulmonary artery acceleration time (PAAT, in pulsed wave Doppler mode; eight to ten measurements performed for each rat), tricuspid annular plane systolic excursion (TAPSE) and heart rate. Data were analyzed with EchoPAC software (GE Healthcare).

\section{Measurement of haemodynamics and tissue preparation}

Rats were anesthetized with $2 \%$ sodium pentobarbital ( $50 \mathrm{mg} / \mathrm{kg}$ i.p.) after exposure to hypoxia for 28 days. Then, the right ventricular systolic pressure (RVSP) was measured through right jugular vein puncture to the right ventricle (RV) with a transducer and was recorded by the PowerLab system (ADInstruments, Australia). Following euthanisation, both the rat lungs and hearts were collected. The weights of the right ventricles (RV) and left ventricle plus septum ( $L V+S)$ were measured separately, and the RV/LV + S weight ratio was determined to indicate right ventricular hypertrophy. Next, the lower lung lobes were sectioned into 4 -mm-thick slices and soaked in a $10 \%$ formalin solution $(\mathrm{pH}=7.4)$. The other tissues were kept at liquid nitrogen for further experiments.

\section{Hematoxylin and eosin (H\&E) staining}

The lung lobes were sliced and embedded in paraffin, and cut into $~ 5-\mu m$-thick sections using microtome. Then, the sections were placed on glass slides, stained with hematoxylin and eosin for morphological analysis, and visualized under an Olympus microscope (Tokyo, Japan). The medial wall thickness was analyzed with ImageJ software (National Institutes of Health, USA) and was expressed by the ratio of medial area to cross sectional area (medial/CSA).

\section{Immunohistochemical analysis}

Paraffin-embedded tissues were cut into $4-\mu \mathrm{m}$-thick sections, and tissue sections were deparaffinized in xylene and rehydrated in alcohol. Sections were heated $5 \mathrm{~min}$ to repair antigenicity in a pressure cooker, treated with $3 \% \mathrm{H}_{2} \mathrm{O}_{2}$ to inactivate endogenous peroxidase activity for $10 \mathrm{~min}$, and incubated with goat serum for 10 min to block nonspecific antibody binding. Sections were incubated with the mouse antiNDRG1 monoclonal antibody $\left(1 / 200\right.$, Santa Cruz, CA) overnight at $4^{\circ} \mathrm{C}$. After incubation with the secondary antibody, the signal was developed with 3, 3'- diaminobenzidine (DAB).

\section{Cell culture}


Human pulmonary arterial endothelial cells (HPAECs) (ScienCell Research Laboratories, USA) were cultured according to the manufacture's instruction. HPAECs (5-8 passages) were cultured in complete endothelial cell medium (ECM) with $5 \%$ fetal bovine serum (FBS) and $1 \%$ Penicillin-Streptomycin solution. Cells were used for experiments at $80-90 \%$ confluence. Cells in the normoxia group were maintained at $37^{\circ} \mathrm{C}$ in $21 \% \mathrm{O}_{2}$ and $5 \% \mathrm{CO}_{2}$ (Thermo Fisher Scientific, USA). Cells in the hypoxia groups were cultured at $37^{\circ} \mathrm{C}$ in $1 \%$ oxygen, $94 \% \mathrm{~N}_{2}$, and $5 \% \mathrm{CO}_{2}$ (Thermo Fisher Scientific, USA).

\section{Establishment of stable cell clones}

Lentiviral vectors for shRNAs targeting NDRG1 or TAF15 and the lentiviral pCDH vector for NDRG1 overexpression were purchased from Addgene (Cambridge, MA, USA). These vectors together with the packaging vectors (Addgene) were transfected into HEK293T cells for preparation of recombinant lentiviruses. HPAECs were infected by lentiviruses in the presence of polybrene $(5 \mu \mathrm{g} / \mathrm{mL})$. Cells were selected for one week using puromycin $(2 \mu \mathrm{g} / \mathrm{mL})$ after infection for $72 \mathrm{~h}$. The selected cell lines were prepared for following experiments.

\section{Proliferation assay}

Proliferation of HPAECs was measured using 5-ethynyl-20-deoxyuridine (EDU) incorporation assay kit (C0075S, Beyotime Biotechnology) and Cell Counting Kit-8 (CCK-8) assay (C0041, Beyotime

Biotechnology), according to the manufacturer's instructions. For the EDU assay, the HPAECs were seeded into 24-well plates at $1 \times 10^{5}$ cells / well and incubated for $24 \mathrm{~h}$ under different conditions. Images were taken under a laser scanning confocal microscope (Olympus, Japan) and the percentage of EDU-positive cells was calculated. For the CCK-8 assay, the HPAECs were seeded into 96 -well plates at $5 \times 10^{3}$ cells / well in complete medium under normoxic conditions. Cells were treated under different conditions, and then the culture medium was removed. A total of $110 \mu \mathrm{L}$ ECM containing CCK-8 (CCK8: ECM $(v / v)=1: 10)$ was added to each well, and the cells were incubated for $4 \mathrm{~h}$. Finally, absorbance at $450 \mathrm{~nm}$ was measured using an Epoch Microplate Spectrophotometer (BioTek, USA).

\section{Endothelial cell migration}

A 24-well transwell plate ( $8 \mu \mathrm{m}$ pore size, Corning, USA) was used to measure cell migration. A total of $1 \times 10^{4}$ cells in $250 \mu \mathrm{L}$ serum-free ECM were placed into the upper chamber, and $500 \mu \mathrm{L}$ ECM containing $10 \%$ FBS was added to the lower chambers. Then the plates were placed in different conditions for $24 \mathrm{~h}$. Cells on the top side of each insert were then scraped off and the wells were fixed in $4 \%$ paraformaldehyde, stained by crystal violet, photographed, and counted under microscope (Olympus, Japan).

\section{Endothelial cell tube formation assays}

Precooled 48-well plates were coated with $150 \mu \mathrm{l}$ of Matrigel (\#354248, Corning, USA) at $37^{\circ} \mathrm{C}$ for $30 \mathrm{~min}$. The HPAECs treated under different conditions were seeded at $1 \times 10^{4}$ cells / well and cultured for $6-8 \mathrm{~h}$. Tube formation was observed under microscope (Olympus, Japan) and the tube lengths were calculated using Image J software (National Institutes of Health, USA). 


\section{Immunofluorescence staining}

The HPAECs treated under different conditions were fixed with $4 \%$ paraformaldehyde, permeabilized with $0.5 \%$ Triton X-100, blocked with donkey serum, and incubated with primary antibodies against NDRG1 or TAF 15 at $4^{\circ} \mathrm{C}$ overnight, followed by incubation with Alexa Fluor-conjugated secondary antibodies (Jackson ImmunoResearch, USA) for $2 \mathrm{~h}$ at $37^{\circ} \mathrm{C}$. Cells were then counterstained with 4囚,6-diamidino-2phenylindole (DAPI). Staining was visualized and photographed via a laser scanning confocal microscope (Olympus, Japan).

\section{Quantitative real-time PCR}

Total RNA was extracted using Trizol reagents (Invitrogen, USA) from lung tissue or cultured HPAECs. Reverse transcription was performed using HiScript II SuperMix reverse transcriptase (Vazyme). cDNA was amplified and detected using Hieff q PCR SYBR Green Master Mix (YEASEN, China). The relative expression level of mRNA was calculated by the $2-\triangle \triangle C T$ method. The qRT-PCR primers were designed and synthesized by Sunny Biotechnology (Shanghai, China), and the primer sequences were shown in supplementary materials.

\section{Nuclear and cytoplasmic protein extraction}

Cell pellets were prepared and treated according to the instructions of a nuclear and cytoplasmic protein extraction kit (Beyotime Biotechnology, China) to isolate nuclear proteins from the cytoplasm. The proteins extracted were assessed by western blotting. $\beta$-actin (Proteintech) and Histone-H3 (CST) were used as loading controls for cytosolic and nuclear proteins, respectively.

\section{Liquid chromatography mass spectrographic (MS) analysis}

To investigate the potential proteins binding to NDRG1, a total of $500 \mu \mathrm{g}$ of cell lysate extracted from HPAECs with Nonidet P 40 (NP-40) buffer and were then centrifuged at $12,000 \times \mathrm{g}$ at $4^{\circ} \mathrm{C}$ for $30 \mathrm{~min}$ to remove cell debris. Five percent of the cell lysates were kept as inputs. The rest was precleared with Protein G Magnetic Beads (Bio-Rad, USA) for $2 \mathrm{~h}$ at $4^{\circ} \mathrm{C}$ and then immunoprecipitated with the corresponding antibodies overnight at $4^{\circ} \mathrm{C}$. The beads were harvested, and the bound proteins were then separated by $10 \%$ SDS-PAGE and stained with mass silver stain (Sangon Biotech Shanghai Co., Ltd.). The proteins were extracted from the gel, re-suspended in $100 \mu \mathrm{l}$ of $\mathrm{ddH}_{2} \mathrm{O}$, and analyzed by mass spectrographic via high-performance liquid chromatography and a Q Exactive Mass Spectrometer (Thermo Scientific, Waltham, Massachusetts) at Shanghai Applied Protein Technology Co., Ltd. The original files were transformed with Proteomics Tools 3.1.6 software, and Mascot 2.2 software was used for database screening.

\section{Coimmunoprecipitation (CO-IP) and immunoblotting}

Cells were lysed in Nonidet P 40 (NP-40) buffer (1 mM PMSF) for $1 \mathrm{~h}$ on ice, and were then centrifuged at $12,000 \times \mathrm{g}$ at $4^{\circ} \mathrm{C}$ for $30 \mathrm{~min}$ to remove cell debris. Five percent of the cell lysates were kept as inputs. The rest was precleared with Protein G Magnetic Beads (Bio-Rad, USA) for $2 \mathrm{~h}$ at $4^{\circ} \mathrm{C}$ and then 
immunoprecipitated with the corresponding antibodies overnight at $4^{\circ} \mathrm{C}$. The beads were harvested, and the bound proteins were resolved by SDS-PAGE and analyzed by Western blotting. For Western blotting, cells were lysed in radio RIPA buffer for $1 \mathrm{~h}$ on ice, and centrifuged at $12,000 \times \mathrm{g}$ at $4^{\circ} \mathrm{C}$ for $30 \mathrm{~min}$ to remove cell debris. Protein samples were subjected to SDS-PAGE and transferred onto polyvinylidene fluoride (PVDF) membranes, followed by blocking and probing with the indicated antibodies for detection. Incubation with appropriate secondary antibodies (Jackson Immuno Research Laboratories, USA) was also performed. The chemiluminescence ECL kit (Yeasen, China) was used to detect the protein bands of interest, and band density was quantified by Image $\mathrm{J}$ software (National Institutes of Health, USA). The antibodies used for IP and primary antibodies for Western blotting are as follows: NDRG1 (1:1000, \#sC398291), TAF15 (1:1000, \#CST28409), Notch1 (1:1000, \# sc-376403), $\beta$-actin (1:8000, \#66009-1-lg, ProteinTech, USA), histone-H3 (1:2000, \#4499, CST), and a-tubulin (1:8000, \# ab7291).

\section{Luciferase reporter assay}

HEK293T cells were plated into 24-well plates at a density of $2 \times 10^{4}$ cells per well 1 day before transfection. Transfections were performed using Lipofectamine 3000 (Invitrogen, USA). Cells were cotransfected with the mixture of a firefly luciferase reporter vector, a Renilla luciferase-expressing vector and other plasmids according to the protocols and experimental design. Luciferase activity was assayed using the Dual-Luciferase Reporter Assay System (Yeasen Biotech, China) and a luminometer (Glomax $20 / 20$, Promega) after transfection for $24 \mathrm{~h}$. Firefly luciferase activity was normalized to Renilla luciferase activity. All experiments were performed at least three times.

\section{Bioinformatics analysis}

Raw gene expression data GSE11341 were downloaded from the Gene Expression Omnibus (GEO) (http://www.ncbi.nlm.nih.gov/geo/) of the National Center for Biotechnology Information (NCBI). GSE11341, deposited by Costello CM et al [20], was from the following GPL96 platform: Affymetrix Human Genome U133A Array, containing microvascular endothelial cells $(n=3)$ in normoxia $\left(21 \% \mathrm{O}_{2}\right)$, or hypoxia $\left(1 \% \mathrm{O}_{2}\right)$ for $24 \mathrm{hrs}$ and $48 \mathrm{hrs}$.

We analyzed the change in the mRNA profiles between control HPAECs and those subjected to TAF15 knockdown. Total RNA was isolated and CDNA library preparation was performed according to the manufacturer's instructions. For the QC step, an Agilent 2100 Bioanalyzer and an ABI StepOnePlus RealTime PCR System were used to qualify and quantify the sample library. Each cDNA library was amplified once before sequencing. Sequencing was performed on an Illumina HiSeq X Ten at Biotecan Co., Ltd sequencer (Shanghai, China). Differential expression analysis was performed by using the "limma" $\mathrm{R}$ package; the expression profiles were compared to identify the DEGs. $P$ values and adjusted $P$ values were calculated using t-tests. Genes meeting the following criteria were further analyzed: 1) an absolute log fold change $>1$ and 2 ) an adjusted $P<0.05$. DEGs with log2 (fold-change) $<0$ were considered downregulated, whereas those with log2 (fold-change) $>0$ were considered up-regulated. The heatmap plots for the DEGs were illustrated by using RStudio software. The functional enrichment analysis of DEGs was performed using the database for Annotation, Visualization, and Integrated Discovery (DAVID). Gene 
ontology (GO) including biological process (BP), cellular component (CC) and molecular function (MF) were used to predict protein functions. KEGG (Kyoto encyclopedia of genes and genomes) pathway analysis was used to assign sets of DEGs to specific pathways to enable the construction of the molecular interaction, reaction and relationship networks.

\section{Statistical analysis}

GraphPad Prism software (version 5.01, United States) was used for statistical analyses. Data were presented as the mean \pm standard error of mean (SEM). The two-tailed Student's $t$-test was used for comparisons of two independent groups. One-Way ANOVA was used to evaluate the statistical significance among three or more groups. Two-tailed $P$ values $<0.05$ were considered statistically significant.

\section{Results}

\section{NDRG1 was highly expressed in endothelial cells under hypoxia}

To investigate the molecular mechanism of endothelial dysfunction during hypoxia, we utilized the GEO database to screen for genes differentially expressed in microvascular endothelial cells $(n=3)$ under normoxia $\left(21 \% \mathrm{O}_{2}\right)$ or hypoxia $\left(1 \% \mathrm{O}_{2}\right)$ (Dataset No. GSE11341). One hundred and thirty-eight genes (Supplementary materials) and 111 genes (Supplementary materials) were obtained after assessing the RNA-seq data in hypoxic exposure for $24 \mathrm{hrs}$ and $48 \mathrm{hrs}$, respectively. Of note is NDRG1, which, albeit known to be hardly detectable in endothelial cells, was significantly upregulated in both 24 and 48hrs hypoxia at the RNA level (Fig. 1A, B). Consistently, NDRG1 was highly expressed in cultured HPAECs under hypoxia but not normoxia at both mRNA and protein levels (Fig. 1C, D). Further cellular immunofluorescent staining verified that NDRG1 was mainly expressed in both the nucleus and the cytoplasm (Fig. 1E). These results revealed that NDRG1 expression is induced by hypoxic exposure timedependently in HPAECs.

\section{NDRG1 was highly expressed in HPH lung samples}

We further examined NDRG1 expression in lung homogenates from HPH patients and rat models. In contrast to control donors or animals, NDRG1 was significantly upregulated in the lung of HPH patient and rats (Fig. 2A-D). To further confirm this result, we performed immunohistochemistry in the same tissue sample section. A strong immunoreactivity of NDRG1 was observed in the intimal layer of pulmonary arteries of subjects with HPH compared with those from normal donors ( $n=3$ for each), as well as in those of HPH model rats when compared with normal rats ( $n=6$ for each) (Fig. 2E). These data suggest that NDRG1 might play a regulatory role in the pathogenesis of $\mathrm{HPH}$.

\section{Effect of NDRG1 knockdown and overexpression on proliferation, migration and angiogenic response of HPAECs}


To explore the role of NDRG1 in endothelial dysfunction, we firstly knocked down endogenous NDRG1 in HPAECs through infection with lentiviruses containing three specific shRNAs (Supplementary Figure S1, A-D). The desired gene knockdown was achieved in hypoxia-exposed cells after expression of NDRG1targeted (NDRG1-KD2 and NDRG1-KD3) but not control (NDRG1-NC) shRNAs (Supplementary Figure S1, $B-D)$. We next evaluated the effects of NDRG1 silencing on the proliferation of HPAECs via EDU and CCK8 assays. The results showed that NDRG1 knockdown decreased the ratios of EDU-positive HPAECs and the viability of cells (Fig. 3A-B). Transwell assays showed that it also reduced the migration of HPAECs (Fig. 3C). Finally, tube formation assays were performed to evaluate the effect of NDRG1 deficiency on angiogenesis. As a result, NDRG1 knockdown significantly decreased branches numbers and tubule length of HPAECs (Fig. 3D). We next examined the effect of NDRG1 overexpression on proliferation, migration and angiogenic response of HPAECs. Abundant gene expression was observed after lentivirusbased delivery of NDRG1 (Supplementary Figure S2 A-D). NDRG1 overexpression led to a substantial increase in proliferation, migration and angiogenic response of HPAECs (Supplementary Figure S3 A-H). Taken together, these results clearly demonstrated that NDRG1 promotes the proliferation, invasion and tube formation of HPAECs.

\section{NDRG1 knockdown alleviates hypoxia-induced PH and right ventricular hypertrophy}

To further validate its role in hypoxia-induced PH, we knocked down NDRG1 in normoxia (N4W) or chronic hypoxia (H4W) exposed rats via i.v. administration of the shRNAs (Supplementary Figure S4, A and B). The results showed that, while hypoxia significantly increased the RVSP and RV/LV + S weight ratio of rats, this was significantly relieved by knockdown of NDRG1 (Fig. 4A, B). Moreover, hypoxia induced $<100$ $\mu \mathrm{m}$ intrapulmonary arteriole remodelling, while NDRG1 knockdown alleviated hypoxia-induced pulmonary vascular remodeling, as evidenced by decreased pulmonary vascular wall thickness and muscularization, and the ratio of medial area to cross sectional area (medial/CSA) (Fig. 4C-D). Echo-Doppler scans showed greater PAATs and a smaller TAPSEs in hypoxia-exposed rats subjected to NDRG1 knockdown than in those injected with a control shRNA (Fig. 4E-F), although no difference was observed between control and NDRG1 knockdown groups in rats raised in normoxic conditions. These results confirmed the promoting role of NDRG1 in pathogenesis of HPH by regulating haemodynamic changes and pulmonary vascular remodeling.

\section{NDRG1 directly interacts with TAF15 and regulates the subcellular localization of TAF15}

To explore the potential mechanism by which NDRG1 regulates the proliferation, invasion and angiogenesis of HPAECs, we performed immunoprecipitation (IP) assay to explore the potential binding protein of NDRG1. Further mass spectrographic analysis indicated that TATA-binding protein-associated factor 15 (TAF15), a component of the RNA polymerase II, was among the candidate proteins binding to NDRG1 (Fig. 5A). Co-IP verified the interaction between NDRG1 and TAF15 in HPAECs (Fig. 5B). Immunofluorescence staining results showed that NDRG1 co-localized with TAF15 in the nuclei of 
HPAECs (Fig. 5C). Since NDRG1 knockdown or overexpression failed to affect the protein levels of TAF15 (Supplementary Figure S5, A-B), we explored whether the subcellular localization of TAF15 was regulated by NDRG1. Indeed, Western blot analysis of separated nuclear and cytoplasmic proteins showed that the levels of TAF15 increased in the cytoplasm and decreased in the nucleus after NDRG1 knockdown (Fig. 6A-B), while NDRG1 overexpression promoted the nuclear localization of TAF15 decreased in the cytoplasm and increased in the nucleus after (Supplementary Figure S6, A-B). The regulation of subcellular localization of TAF15 by NDRG1 was further verified by immunofluorescence staining (Fig. 6E). Thus, NDRG1 binding facilitates nuclear accumulation of TAF15 in hypoxic HPAECs.

\section{NDRG1 regulates endothelial dysfunction through TAF15}

Based on the above results, we speculated that NDRG1 may regulate endothelial dysfunction through TAF15. To test this hypothesis, we knocked down TAF15 in HPAECs (Supplementary Figure S7 A-B) and performed EDU and CCK-8 assays, transwell assays and tube formation assays. TAF15 silencing counteracted the proliferation and migration of HPAECs induced by NDRG1 overexpression (Fig. 7A-C). TAF15 knockdown also abrogated the effect of NDRG1 on in vitro tube formation of HPAECs, as evidenced by reduced branches and tubule length of HPAECs (Fig. 7D). Taken together, these results indicated that NDRG1 causes endothelial dysfunction through inducing nuclear translocation and activation of TAF15.

\section{Notch1 is downstream of TAF15 in the regulation of endothelial function}

To further explore the downstream signalling pathway of TAF15 in HPAECs, we silenced TAF15 and performed RNA-seq to screen for differentially expressed genes (DEGs). The DEGs were then analyzed by Gene Ontology (GO) and Kyoto Encyclopedia of genes and genomes (KEGG) pathway enrichment analysis. Go analysis showed that TAF15 may be involved in the regulation of cell migration, angiogenesis and proliferation (Supplementary Figure S8, A-C). KEGG analysis showed that TAF15 may play a role by regulating PI3K-Akt, p53, HIF-1 and Notch signaling pathways (Fig. 8A). Further RT-PCR and western blot analysis showed that TAF15 knockdown impaired the expression of Notch1 (Fig. 8B-D), suggesting that TAF15 might lead to endothelial dysfunction and HPH through Notch1. Consistent with the presence of a predicted TAF1 5 binding site in the Notch1 promoter, a dual luciferase assay showed that TAF15 overexpression in HEK293T cells transfected with the pGL3 construct of Notch1 promoter significantly increased the luciferase activity, which was not observed in those transfected with a construct of Notch1 promoter with mutations in the putative TAF15 binding site (MUT) (Fig. 8E). Therefore, TAF15 activation is likely to impair endothelial homeostasis through regulation of Notch1.

\section{Discussion}

In the present study, our investigation using HPAECs, HPH rat models and clinical specimens led to several key findings. Firstly, Hypoxia exposure of HPAECs induces a redundant expression of NDRG1 in a time-dependent manner. Secondly, NDRG1 expression is increased in lungs and pulmonary arteries of 
$\mathrm{HPH}$ patients and HPH model rats. Thirdly, NDRG1 positively regulates proliferation, migration and angiogenic response of HPAECs via TAF15, and inhibition of NDRG1 in vivo attenuated right ventricular systolic pressure and right ventricular hypertrophy of HPH rats. Lastly, NDRG1 can promote the nuclear localization of TAF15 and subsequently the upregulation of Notch1, although the precise role of TAF15/Notch1 in HPH remains to be determined.

Omics technology holds great promise to identify new biomarkers and thereby improve the diagnosis and treatment of PH [21]. In this study, we searched a GEO dataset (GSE11341) [20], and found that NDRG1, known to be absent in normal endothelial cells, was highly expressed in endothelial cells stimulated by hypoxia, indicating that NDRG1 may be involved in the response to acute and chronic hypoxia in endothelial cells. As a gene related to hypoxia stress, NDRG1 could be transcriptionally activated by HIF$1 \mathrm{a}$ and ETS proto-oncogene 1 (ETS1) [22]. HIF-1 was reported drive the initial response to acute hypoxia (2-24h) and HIF-2 is a key player in the chronic response (48-72h) [23]. Our study found that NDRG1 is still highly expressed after hypoxia exposure for 24 hours, suggesting that NDRG1 may be regulated by both HIF-1a and HIF-2a. Nontheless, further studies are required to determine how NDRG1 is upregulated in HPAECs during the pathogenesis of $\mathrm{HPH}$.

The involvement of NDRG1 in HPH was demonstrated by our studies, which showed an increased level of NDRG1 in pulmonary vasculature of HPH patients, as well as in HPH model rats. Additionally, we observed that increased NDRG1 expression occurred in the endothelium of HPH vessels compared to donor vessels, suggesting that NDRG1 might be involved in endothelial dysfunction. Further study confirmed that NDRG1 positively regulates proliferation, migration and angiogenic response of HPAECs, and inhibition of NDRG1 in vivo attenuated right ventricular systolic pressure and right ventricular hypertrophy of HPH rats. NDRG1 plays multifunctional roles in cell growth, development, differentiation, stress response of various cells including the endothelial cells $[17,18]$. Knockdown of NDRG1 decreased haemangioma endothelial cell proliferation and downregulated the c-MYC oncoprotein [24]. Ablation of Serum and glucocorticoid inducible kinase 1 (SGK1) caused decreased NDRG1 phosphorylation, defective endothelial cell migration and tube formation in vitro, and impaired angiogenesis in vivo after myocardial infarct [25]. Repression of NDRG1 decreased migration of endothelial cells and impaired neoangiogenesis under intermittent hypoxia conditions [26]. In addition, NDRG1 deficiency in endothelial cell prevented aorta angiogenesis and the activation of phospholipase CY1 (PLCY1) and ERK1/2 by VEGF$A$ [27]. Consistent with these observations, our findings in HPAECs suggest an essential role of NDRG1 in the development of $\mathrm{HPH}$.

Our immunoprecipitation assay and mass spectrographic analysis indicated that NDRG1 could bind with TAF15, and immunofluorescence results showed NDRG1 co-localized with TAF15 in the nucleus of HPAECs. TAF15 belongs to member of the TET family of RNA-binding proteins, and previous studies have found that TAF15 showed both cytoplasmic and nuclear expression in almost all human tissues [28]. We found here that NDRG1 regulates the subcellular localization of TAF15 in HPAECs. Previous studies reported that NDRG1 was highly upregulated by Kaposi's sarcoma-associated herpesvirus in the nuclei of endothelial cells nucleus, forming a complex as a scaffold protein [29]. Previous investigations also 
identified the arginine and glycine rich (RGG) domains of TAF15 responsible for the subcellular localisation or shuttle between the nucleus and the cytoplasm [30]. It is thus worth investigating whether NDRG1 promotes nuclear accumulation of TAF15 through direct interaction with the RGG domain in HPAECS.

As a transcription factor and a component of RNA polymerase II, TAF15 licenses the transcription of a large cohort of genes. Hence we conducted RNA-seq assay to detect genes affected by TAF15 knockdown in HPAECs. GO and KEGG analysis showed that TAF15 is enriched in pathways related to proliferation and migration. In particular, we found Notch1, the core molecule of Notch signaling pathway, was downregulated in TAF15-KD HPAECs. Luciferase assay showed that TAF15 could bind the Notch1 promoter and transcriptionally activate the expression of Notch1. Notch signaling pathway plays a critical role in the development and homeostasis of the cardiovascular system [31]. Several studies have linked Notch1 to vascular development and injury, for example, Notch1 knockout and Cre-mediated deletion of endothelial Notch 1 in mice resulted in embryonic lethality [32]. Heterozygous Notch $1^{+/-}$mice showed decreased neointima formation compared with the wild-type littermates after carotid artery ligation [33]. Several reports have further linked Notch1 to pulmonary hypertension [34], since upregulated expression of Notch1 has been found in $\mathrm{PH}$ patients and $\mathrm{PH}$ model animals [35, 36]. Notch1 expression was increased under hypoxia in HPAECs, and Notch1 knockdown inhibited hypoxia-induced proliferation and migration of these cells [36]. Kimberly et al demonstrated that hypoxia-induced activation of Notch signaling mediates HPH via functional activation and upregulation of TRPC6 channels [37]. Similarly, Notch1 activation was found to promote the proliferative phenotype of endothelial cells [38]. In animal experiments, $\gamma$-secretase inhibitor and atorvastatin can effectively suppress the expression of Notch1, hence improving right ventricular systolic pressure and right heart function of rats [36, 39]. In line with these reports, our finding that Notch1 was upregulated by NDRG1/TAF15 in hypoxic HPAECs has added weight to the critical involvement of the canonical Notch pathway in $\mathrm{HPH}$.

\section{Conclusions}

In summary, our work suggests that hypoxia-induced NDRG1 contributes to endothelial dysfunction and $\mathrm{HPH}$ pathogenesis through nuclear localization of TAF15 and transcriptional activation of Notch1. Targeting NDRG1 and downstream signaling may be a promising therapeutic strategy for HPH.

\section{Abbreviations}

The following abbreviations are used in this manuscript: 


\begin{tabular}{|c|c|}
\hline BP & Biological process \\
\hline CC & Cellular component \\
\hline CCK-8 & Cell counting kit-8 \\
\hline CO-IP & Coimmunoprecipitation \\
\hline COPD & Chronic obstructive pulmonary disease \\
\hline CPFE & Combined pulmonary fibrosis and emphysema \\
\hline CSA & Cross sectional area \\
\hline DAVID & Database for Annotation, Visualization, and Integrated Discovery \\
\hline ECM & Endothelial cell medium \\
\hline GEO & Gene expression omnibus \\
\hline GO & Gene ontology \\
\hline HPAECs & Human pulmonary arterial endothelial cells \\
\hline $\mathrm{HPH}$ & Hypoxia-induced pulmonary hypertension \\
\hline ILD & Interstitial lung disease \\
\hline KEGG & Kyoto encyclopedia of genes and genomes \\
\hline LV+S & Left ventricle plus septum \\
\hline MF & Molecular function \\
\hline mPAP & Mean pulmonary arterial pressure \\
\hline MS & Mass spectrographic \\
\hline $\mathrm{NCBI}$ & National Center for Biotechnology Information \\
\hline NDRG1 & $\mathrm{N}$-myc downstream regulated gene- 1 \\
\hline PAAT & Pulmonary artery acceleration time \\
\hline $\mathrm{PH}$ & Pulmonary hypertension \\
\hline PVDF & Polyvinylidene fluoride \\
\hline RV & Right ventricle \\
\hline RVSP & Right ventricular systolic pressure \\
\hline shRNAs & Short hairpin rnas \\
\hline TAF15 & TATA-box binding protein associated factor 15 \\
\hline TAPSE & Tricuspid annular plane systolic excursion \\
\hline
\end{tabular}




\section{Declarations}

\section{Acknowledgements}

The authors would like to express their gratitude to Dr. Lintao Jia in the Fourth Military Medical University for his expert advice and technical assistance.

\section{Authors' contributions}

Conceptualization, SQL; data curation, CWL, LD and NZ; formal analysis, XJZ and RY and GW; investigation, XMW, JL and JWX; writing-original draft preparation, CWL and JZL; writing-review and editing, SHL and YZZ; project administration, JW and DBZ; funding acquisition, SQL. All authors have read and agreed to the published version of the manuscript.

\section{Funding}

This study was supported by the National Natural Science Foundation of China (No.81970048).

\section{Availability of data and materials}

The data used to support the current study are available from the corresponding author on reasonable request.

\section{Ethics approval and consent to participate}

The study was approved by the Research Ethics Committee of Huashan Hospital, Fudan University (KY2016-396), and the experimental procedures were conducted in accordance with the Ethics Committee of Experimental Research at Fudan University Shanghai Medical College.

\section{Consent for publication}

Not applicable.

\section{Competing interests}

The authors declare that they have no competing interests.

\section{Author details}

Department of Pulmonary and Critical Care Medicine, Huashan Hospital, Fudan University, Shanghai, 200040, China.

\section{References}


1. Galiè N, Humbert M, Vachiery JL, et al. 2015 ESC/ERS Guidelines for the diagnosis and treatment of pulmonary hypertension: The Joint Task Force for the Diagnosis and Treatment of Pulmonary Hypertension of the European Society of Cardiology (ESC) and the European Respiratory Society (ERS): Endorsed by: Association for European Paediatric and Congenital Cardiology (AEPC), International Society for Heart and Lung Transplantation (ISHLT). European heart journal. 2016;37(1):67-119.

2. [Chinese guidelines for the diagnosis and treatment of pulmonary hypertension 2018]. Zhonghua xin xue guan bing za zhi. 2018;46(12):933-64.

3. Olschewski $\mathrm{H}$, Behr J, Bremer $\mathrm{H}$, et al. Pulmonary hypertension due to lung diseases: Updated recommendations from the Cologne Consensus Conference 2018. International journal of cardiology. 2018;272s:63-8.

4. Klinger JR. Group III Pulmonary Hypertension: Pulmonary Hypertension Associated with Lung Disease: Epidemiology, Pathophysiology, and Treatments. Cardiology clinics. 2016;34(3):413-33.

5. Heresi GA, Platt DM. Healthcare burden of pulmonary hypertension owing to lung disease and/or hypoxia. 2017;17(1):58.

6. Chebib N, Mornex JF, Traclet J, et al. Pulmonary hypertension in chronic lung diseases: comparison to other pulmonary hypertension groups. Pulmonary circulation. 2018;8(2):2045894018775056.

7. Rowan SC, Keane MP, Gaine S, McLoughlin P. Hypoxic pulmonary hypertension in chronic lung diseases: novel vasoconstrictor pathways. The Lancet Respiratory medicine. 2016;4(3):225-36.

8. Hoeper MM, McLaughlin VV, Dalaan AM, et al. Treatment of pulmonary hypertension. The Lancet Respiratory medicine. 2016;4(4):323-36.

9. Shimoda LA, Laurie SS. Vascular remodeling in pulmonary hypertension. Journal of molecular medicine (Berlin, Germany). 2013;91(3):297-309.

10. Thenappan T, Ormiston ML, Ryan JJ, Archer SL. Pulmonary arterial hypertension: pathogenesis and clinical management. BMJ (Clinical research ed). 2018;360:j5492.

11. Stenmark KR, Fagan KA, Frid MG. Hypoxia-induced pulmonary vascular remodeling: cellular and molecular mechanisms. Circulation research. 2006;99(7):675-91.

12. Huertas A, Guignabert C. Pulmonary vascular endothelium: the orchestra conductor in respiratory diseases: Highlights from basic research to therapy. 2018;51(4).

13. Ranchoux B, Harvey LD, Ayon RJ, et al. Endothelial dysfunction in pulmonary arterial hypertension: an evolving landscape (2017 Grover Conference Series). Pulmonary circulation. 2018;8(1):2045893217752912.

14. Tu L, Dewachter L, Gore B, et al. Autocrine fibroblast growth factor-2 signaling contributes to altered endothelial phenotype in pulmonary hypertension. American journal of respiratory cell and molecular biology. 2011;45(2):311-22.

15. Ma C, Li Y, Ma J, et al. Key role of 15-lipoxygenase/15-hydroxyeicosatetraenoic acid in pulmonary vascular remodeling and vascular angiogenesis associated with hypoxic pulmonary hypertension. Hypertension (Dallas, Tex : 1979). 2011;58(4):679-88. 
16. Lachat P, Shaw P, Gebhard S, et al. Expression of NDRG1, a differentiation-related gene, in human tissues. Histochemistry and cell biology. 2002;118(5):399-408.

17. Fang BA, Kovačević Ž, Park KC, et al. Molecular functions of the iron-regulated metastasis suppressor, NDRG1, and its potential as a molecular target for cancer therapy. Biochimica et biophysica acta. 2014;1845(1):1-19.

18. Park KC, Paluncic J, Kovacevic Z, Richardson DR. Pharmacological targeting and the diverse functions of the metastasis suppressor, NDRG1, in cancer. Free radical biology \& medicine. 2020;157:154-75.

19. Li Y, Yang L, Dong L, et al. Crosstalk between the Akt/mTORC1 and NF-KB signaling pathways promotes hypoxia-induced pulmonary hypertension by increasing DPP4 expression in PASMCs. Acta pharmacologica Sinica. 2019;40(10):1322-33.

20. Costello CM, Howell K, Cahill E, et al. Lung-selective gene responses to alveolar hypoxia: potential role for the bone morphogenetic antagonist gremlin in pulmonary hypertension. American journal of physiology Lung cellular and molecular physiology. 2008;295(2):L272-84.

21. Hemnes AR. Using Omics to Understand and Treat Pulmonary Vascular Disease. Frontiers in medicine. 2018;5:157.

22. Wang Q, Li LH, Gao GD, et al. HIF-1 a up-regulates NDRG1 expression through binding to NDRG1 promoter, leading to proliferation of lung cancer A549 cells. Molecular biology reports. 2013;40(5):3723-9.

23. Koh MY, Powis G. Passing the baton: the HIF switch. Trends in biochemical sciences. 2012;37(9):364-72.

24. Byun JW, An HY, Yeom SD, et al. NDRG1 and FOXO1 regulate endothelial cell proliferation in infantile haemangioma. Experimental dermatology. 2018;27(6):690-3.

25. Zarrinpashneh E, Poggioli T, Sarathchandra P, et al. Ablation of SGK1 impairs endothelial cell migration and tube formation leading to decreased neo-angiogenesis following myocardial infarction. PloS one. 2013;8(11):e80268.

26. Toffoli S, Delaive E, Dieu M, et al. NDRG1 and CRK-I/II are regulators of endothelial cell migration under Intermittent Hypoxia. Angiogenesis. 2009;12(4):339-54.

27. Watari $\mathrm{K}$, Shibata T, Fujita $\mathrm{H}$, et al. NDRG1 activates VEGF-A-induced angiogenesis through PLCY1/ERK signaling in mouse vascular endothelial cells. Communications biology. 2020;3(1):107.

28. Andersson MK, Ståhlberg A, Arvidsson Y, et al. The multifunctional FUS, EWS and TAF15 protooncoproteins show cell type-specific expression patterns and involvement in cell spreading and stress response. BMC cell biology. 2008;9:37.

29. Zhang F, Liang D, Lin X, et al. NDRG1 facilitates the replication and persistence of Kaposi's sarcomaassociated herpesvirus by interacting with the DNA polymerase clamp PCNA. 2019;15(2):e1007628.

30. Jobert L, Argentini M, Tora L. PRMT1 mediated methylation of TAF15 is required for its positive gene regulatory function. Experimental cell research. 2009;315(7):1273-86. 
31. Hofmann JJ, Iruela-Arispe ML. Notch signaling in blood vessels: who is talking to whom about what? Circulation research. 2007;100(11):1556-68.

32. Krebs $L T, X u e Y$, Norton $C R$, et al. Notch signaling is essential for vascular morphogenesis in mice. Genes \& development. 2000;14(11):1343-52.

33. Li Y, Takeshita K, Liu PY, et al. Smooth muscle Notch1 mediates neointimal formation after vascular injury. Circulation. 2009;119(20):2686-92.

34. Babicheva A, Yuan JX. Endothelial Notch1 in Pulmonary Hypertension. Circulation research. 2019;124(2):176-9.

35. Qiao L, Xie L, Shi K, et al. Notch signaling change in pulmonary vascular remodeling in rats with pulmonary hypertension and its implication for therapeutic intervention. PloS one. 2012;7(12):e51514.

36. Dabral S, Tian X, Kojonazarov B, et al. Notch1 signalling regulates endothelial proliferation and apoptosis in pulmonary arterial hypertension. The European respiratory journal. 2016;48(4):1137-49.

37. Smith KA, Voiriot G, Tang H, et al. Notch Activation of $\mathrm{Ca}(2+)$ Signaling in the Development of Hypoxic Pulmonary Vasoconstriction and Pulmonary Hypertension. American journal of respiratory cell and molecular biology. 2015;53(3):355-67.

38. Miyagawa K, Shi M, Chen PI, et al. Smooth Muscle Contact Drives Endothelial Regeneration by BMPR2-Notch1-Mediated Metabolic and Epigenetic Changes. Circulation research. 2019;124(2):21124.

39. Zhu YT, Liu H, Zhang XQ, et al. Effect of atorvastatin on pulmonary hypertension rats through regulating notch signaling pathway. European review for medical and pharmacological sciences. 2020;24(9):5118-26.

\section{Figures}




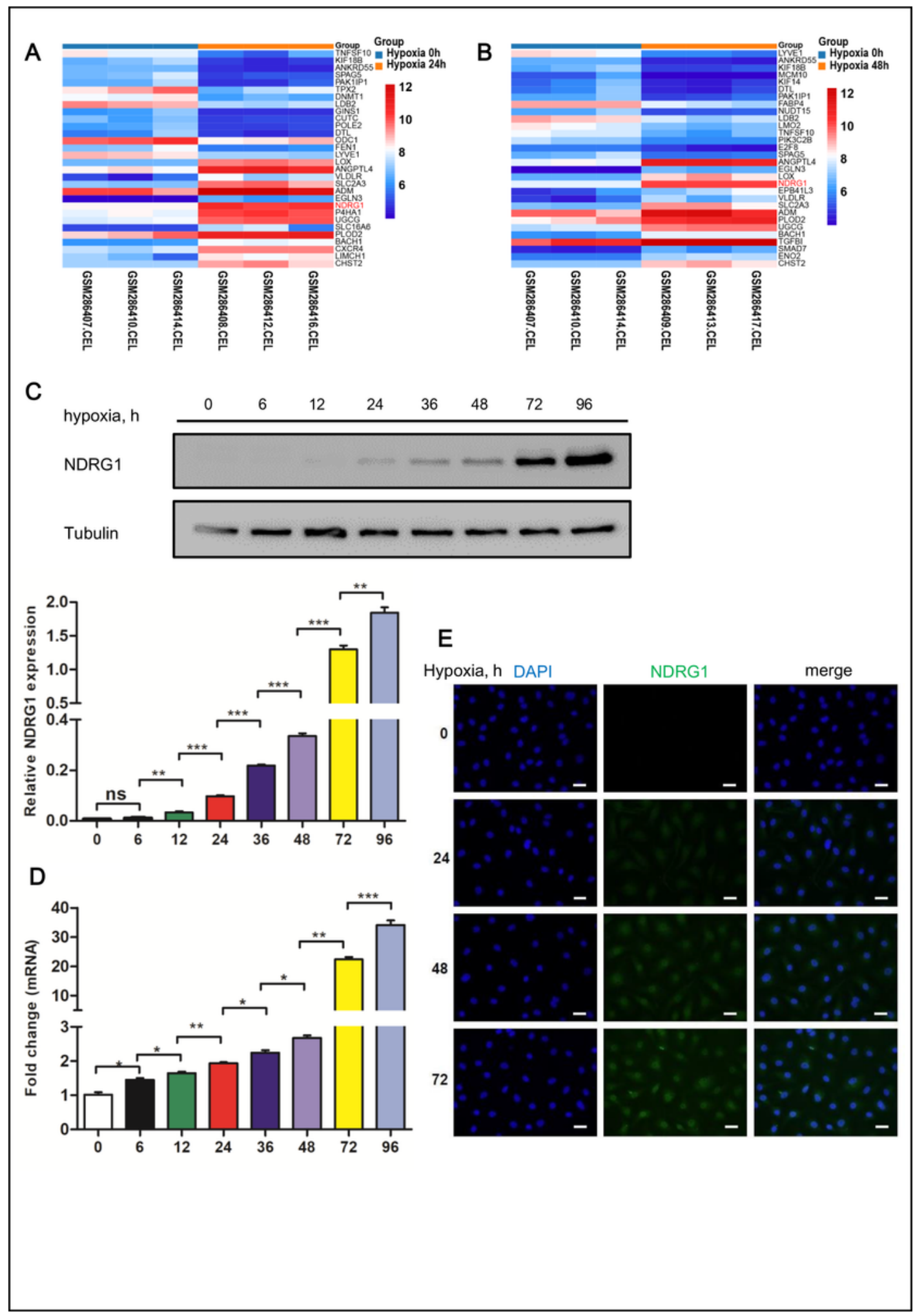

\section{Figure 1}

NDRG1 is a hypoxia-induced gene in human pulmonary arterial endothelial cell (HPAECs). (A, B) Heat maps of differentially expressed genes in HPAECs under hypoxia (1\% 02) for $24 \mathrm{~h}$ (A) and $48 \mathrm{~h}$ (B). (C) Western blotting and densitometric analysis of NDRG1 in HPAECs under hypoxia for 0 to $96 \mathrm{~h}$. Tubulin was blotted as the loading control. ${ }^{\star} p<0.05,{ }^{\star \star} p<0.01$, ${ }^{\star \star *} p<0.001$ versus normoxia. (D) The mRNA levels of NDRG1 was analyzed by real-time PCR, and the fold changes of mRNA levels in cells exposed to 
hypoxia for indicated times versus those in cells cultured under normoxia were plotted. ${ }^{*} p<0.05,{ }^{*} p<$ $0.01, * \star \star p<0.001$. (E) NDRG1 in HPAECs under hypoxia for 0 to $72 \mathrm{~h}$ were detected by immunofluorescence staining. Scale bar, $20 \mu \mathrm{m}$.

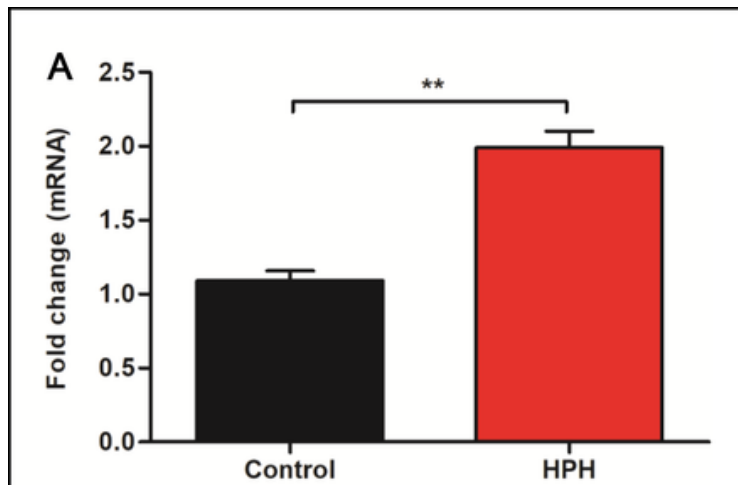

C
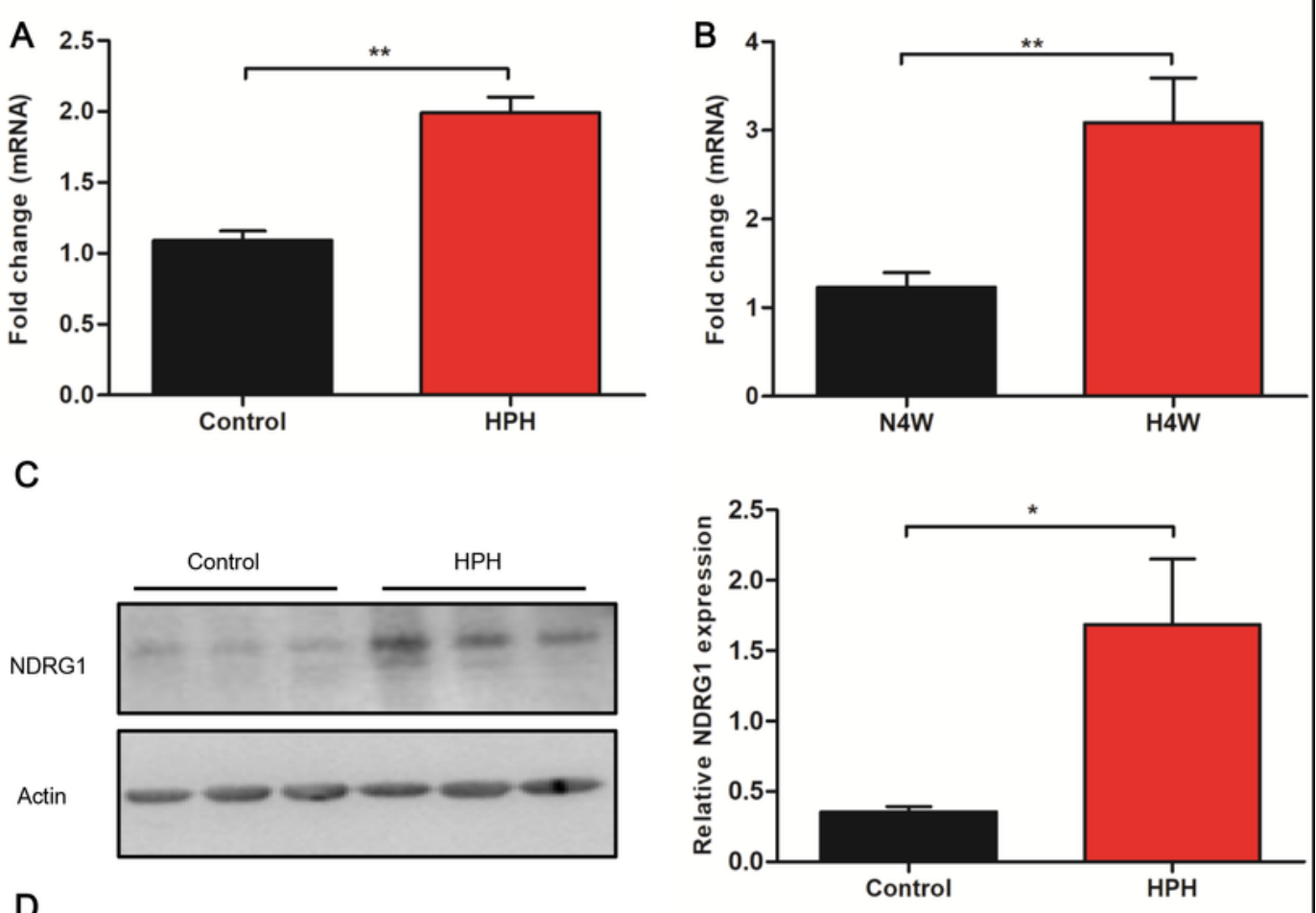

D

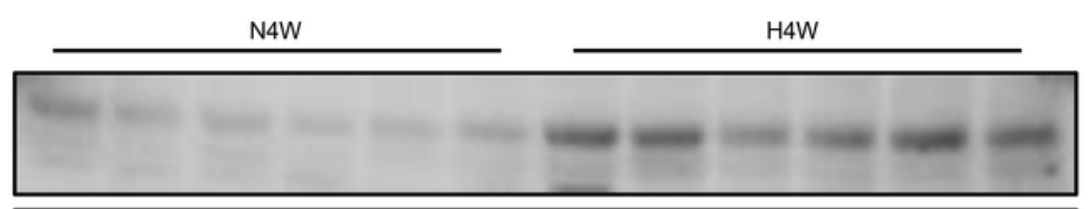

Actin

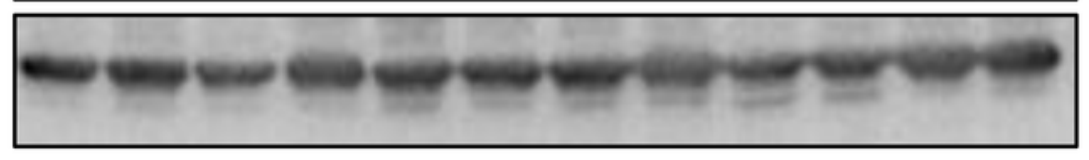

E

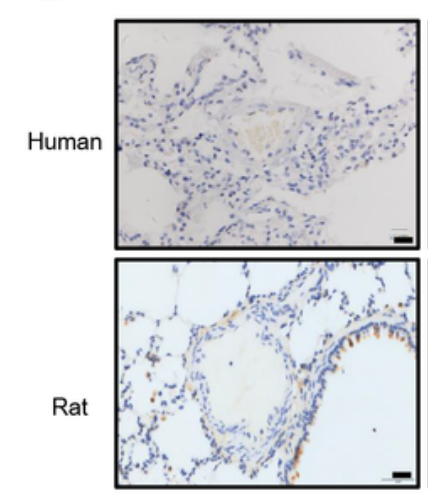

Control

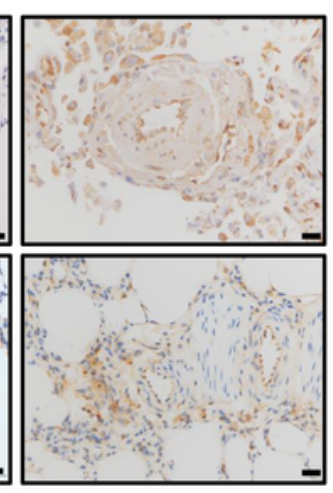

$\mathrm{HPH}$

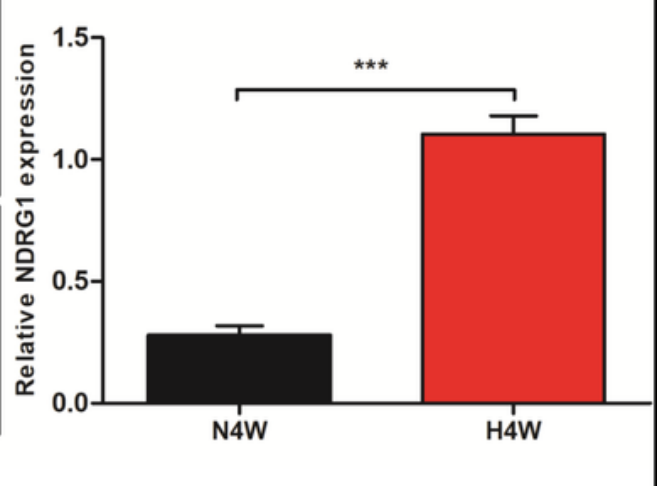

\section{Figure 2}

NDRG1 is highly expressed in pulmonary vessels of HPH patients and model rats. (A) The expression of NDRG1 in lung homogenates of donors and HPH patients was measured by qRT-PCR ( $n=3$, respectively). 
(B) The expression of NDRG1 in lung homogenates of control and HPH model rats was measured by qRTPCR ( $n=6$, respectively). (C) Western blot analysis using lung homogenates of donors and HPH patients $(n=3$, respectively). $\beta$-actin was used as the loading control. (D) Western blot analysis of NDRG1 in lung homogenates of donors and HPH model rats ( $n=6$, respectively). ${ }^{*} p<0.05,{ }^{*} p<0.01,{ }^{* \star *} p<0.001$ versus donors. (E) Representative immunostaining micrographs for NDRG1 using lung sections from non-PH donors or HPH patients, and from control or HPH model rats. Scale bar, $20 \mu \mathrm{m}$.

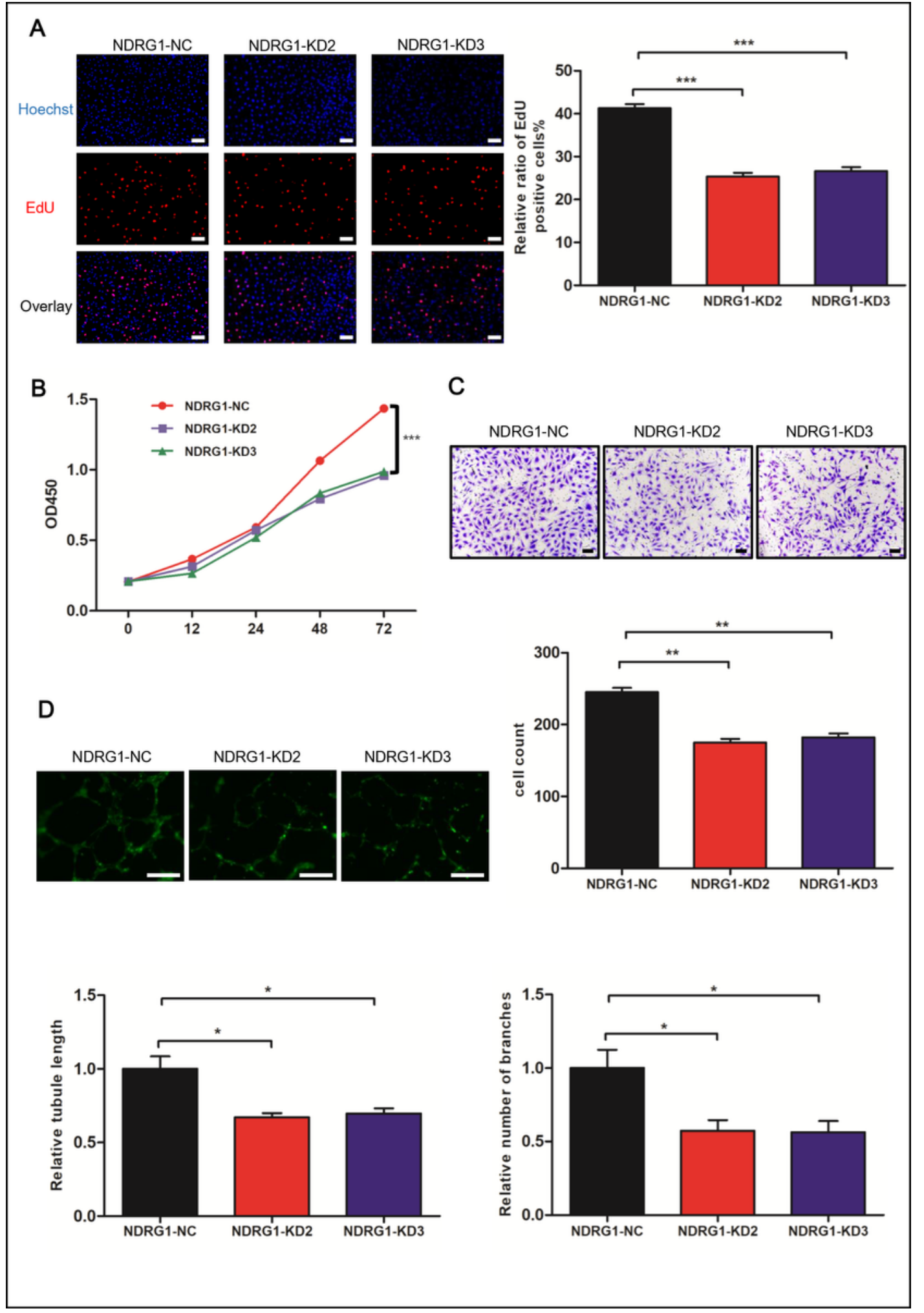

Figure 3 
NDRG1 promotes proliferation, migration and angiogenic response of HPAECs under hypoxia. (A) HPAEC proliferation was measured by EDU assay in control (NDRG1-NC) and NDRG1 knockdown (NDRG1-KD2 and NDRG1-KD3) cells followed by hypoxia exposure. Scale bar, $100 \mu \mathrm{m}$. The ratios of red (EdU) to blue (Hoechst) cells were plotted. (B) Control and NDRG1 knockdown HPAECs were exposed to hypoxia for indicated times and subjected to CCK-8 assay (C) Control and NDRG1 knockdown HPAECs were exposed to hypoxia for $24 \mathrm{hrs}$, and cell migration was measured by transwell assay. Cells migrating to the lower chambers were counted. (D) Control and NDRG1 knockdown HPAECs were exposed to hypoxia for 6-8 hrs, and the angiogenic response was measured by tube formation. The results were shown by relative length of tubules and relative number of branches formed by HPAECs. ${ }^{\star} p<0.05,{ }^{\star \star} p<0.01,{ }^{\star \star \star} p<0.001$. 


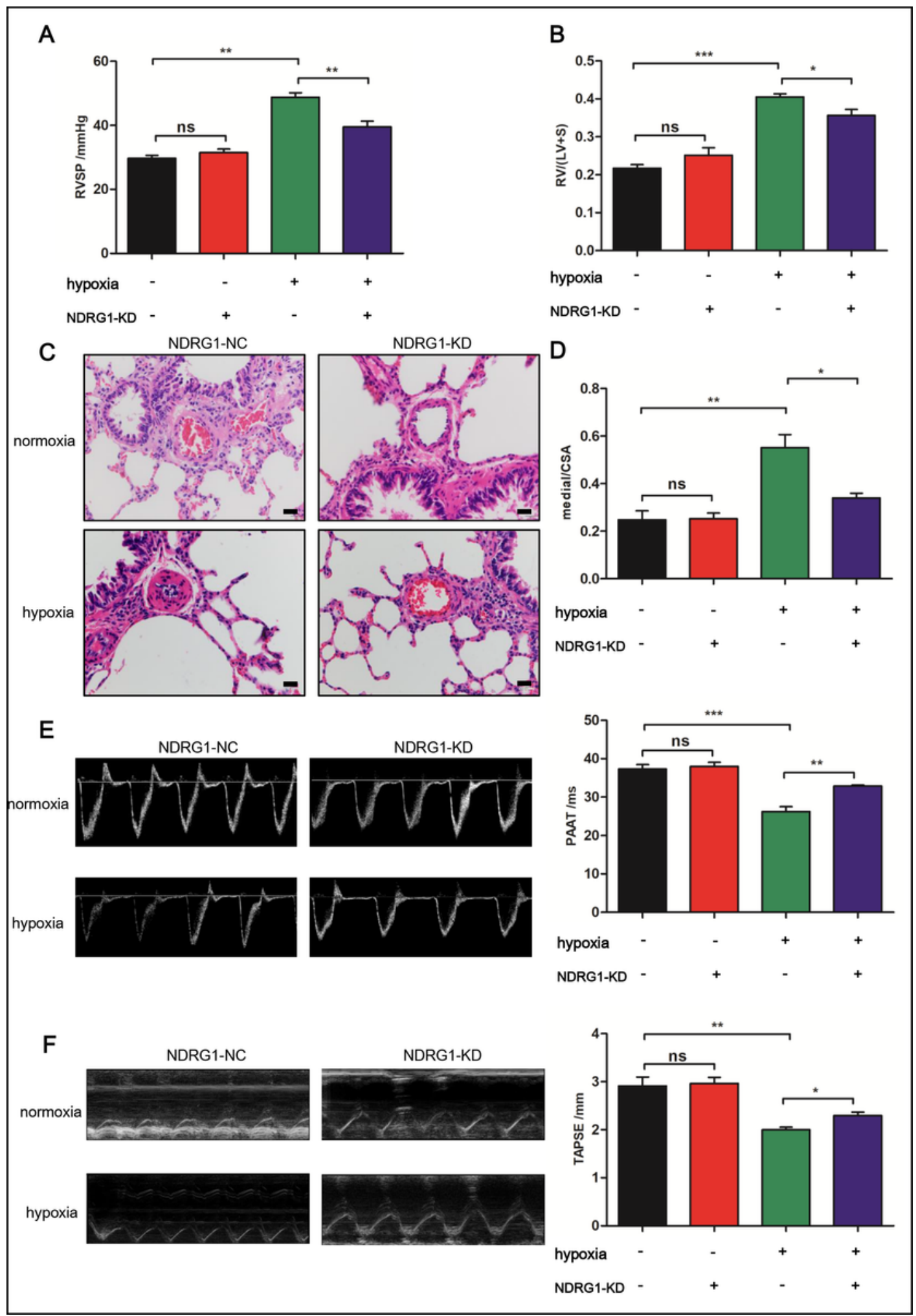

Figure 4

NDRG1 knockdown alleviates hypoxia-induced PH and right ventricular hypertrophy in a rat HPH model. $(A, B)$ A rat model of HPH was generated and treated through i.v. injection of control or NDRG1-targeted shRNAs ( $n=8 /$ group). The RVSP (A) and RV/ (LV+S) (B) were calculated. (C, D) HE staining of paraffinfixed lung sections prepared from rats in (A) for the morphological analysis of pulmonary arteries (C, Scale bar, $20 \mu \mathrm{m})$, followed by calculation of the ratio of medial area to cross sectional area 
(medial/CSA) (D). (E, F) Echocardiography was carried out on rats in (A). Pulmonary arterial acceleration time (PAAT) (E) and tricuspid annular plane systolic excursion (TAPSE) (F) of the rats were monitored and plotted $(n=5)$. The data are expressed as the means \pm standard errors of the mean. ${ }^{*} p<0.05,{ }^{* \star} p<0.01$, $\star \star \star p<0.001$.

A

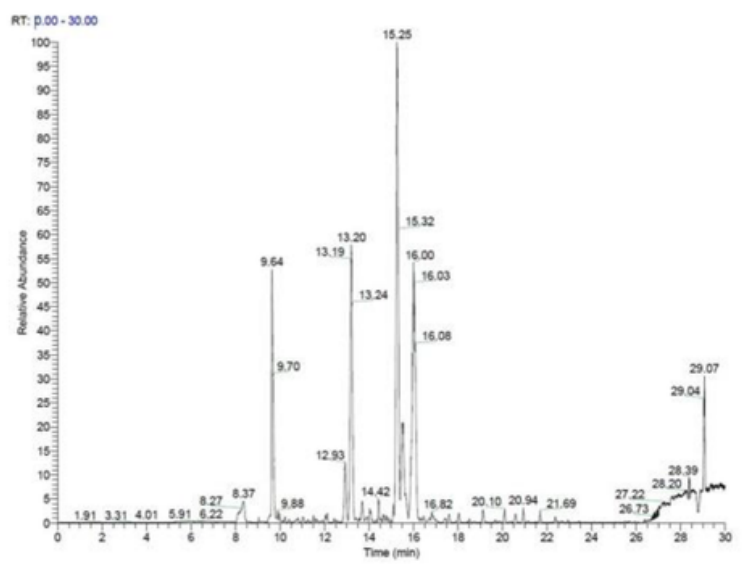

B Input IP: IgG IP: NDRG1

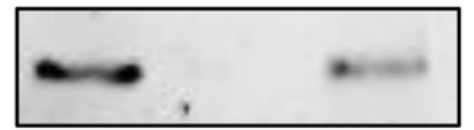

TAF15 Input IP: IgG IP: TAF15

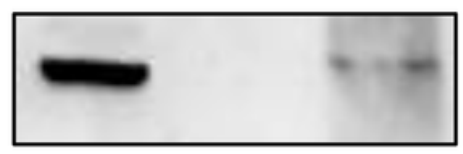
NDRG1

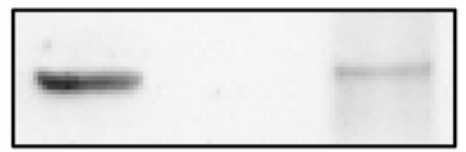
TAF15 NDRG1+TAF15 merge

C

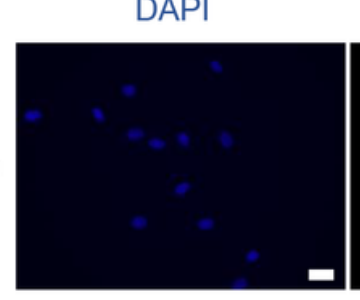

NDRG1
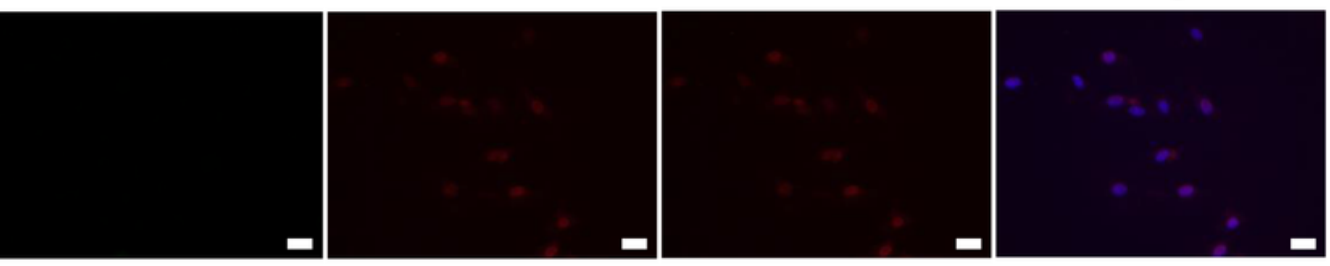

hypoxia
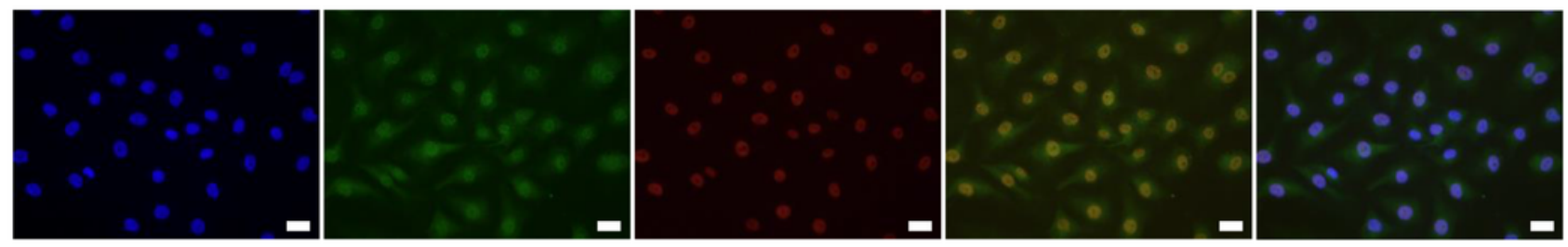

\section{Figure 5}

NDRG1 interacts with TAF15 in HPAECs. (A) The lysates of HPAECs were immunoprecipitated with an NDRG1 antibody, and mass spectrometry analysis was conducted to discover the potential interacting protein. Trypsin-digested fragment detected by mass spectrometry is presented. (B) Lysates of HPAECs 
were subjected to immunoprecipitation and subsequent immunoblotting with the indicated antibodies HPAECs. (C) HPAECs were exposed to normoxia or hypoxia for XX hrs, and were subjected to immunofluorescence staining for NDRG1 (green) and TAF15 (red). The nuclei of cells were counterstained with DAPI (blue). Scale bar, $20 \mu \mathrm{m}$.

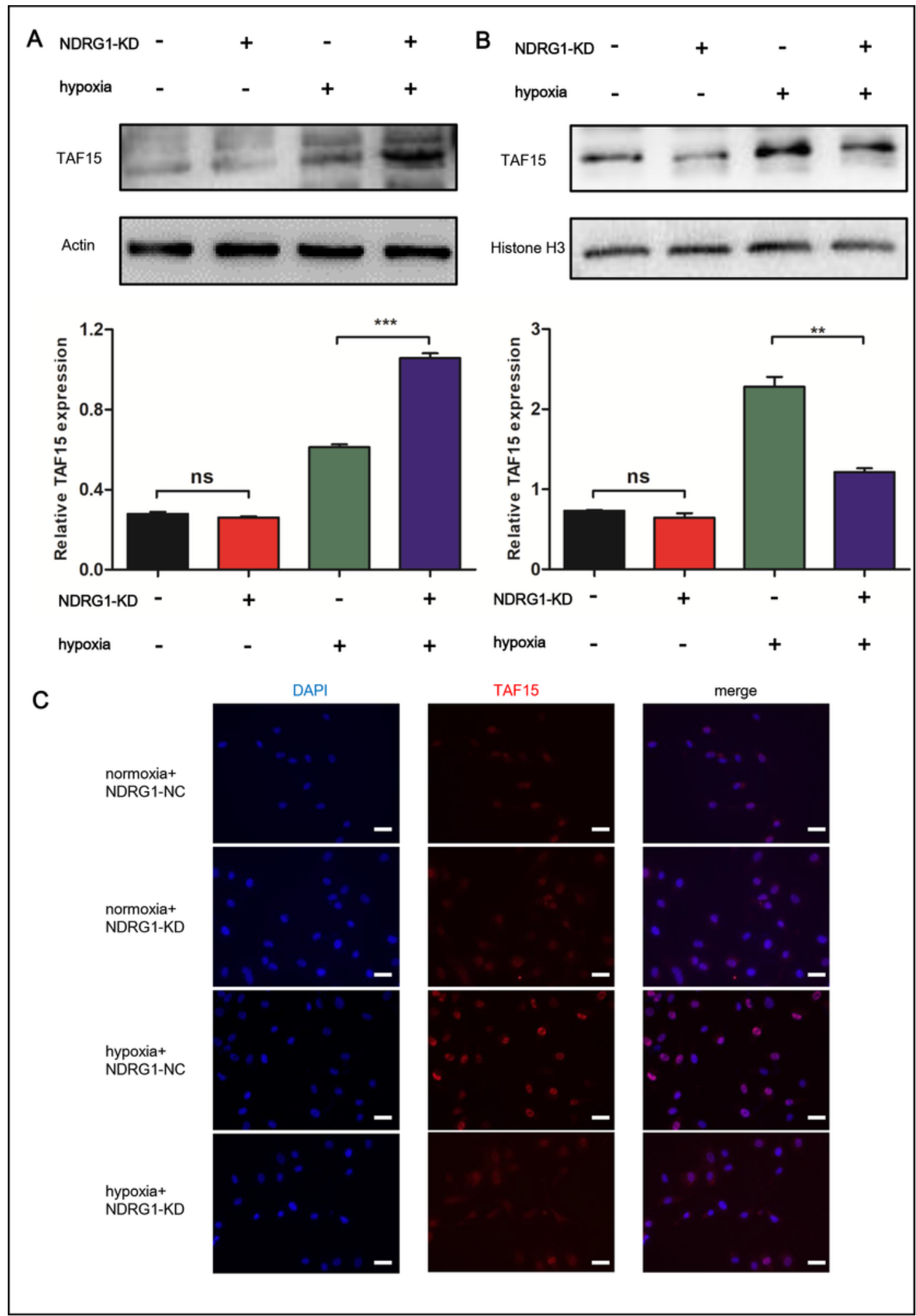

Figure 6 
NDRG1 regulated the subcellular localization of TAF15 in HPAECs. (A, B) HPAECs were subjected to hypoxia exposure and/or knockdown of NDRG1, followed by preparation of cytoplasmic and nuclear extracts of cells. Western blot analysis of the cytoplasmic (A) and nuclear (B) HPAECs proteins was then performed. $\beta$-actin and $\mathrm{H}$ histone $\mathrm{H} 3$ were blotted as the loading control for the cytoplasmic and nuclear proteins, respectively. ${ }^{*} p<0.01,{ }^{* *} p<0.001$. (C) HPAECs were treated as described in (A), and were subjected to immunofluorescence staining for NDRG1 (green) and TAF15 (red). The nuclei of cells were counterstained with DAPI (blue). Scale bar, $20 \mu \mathrm{m}$.

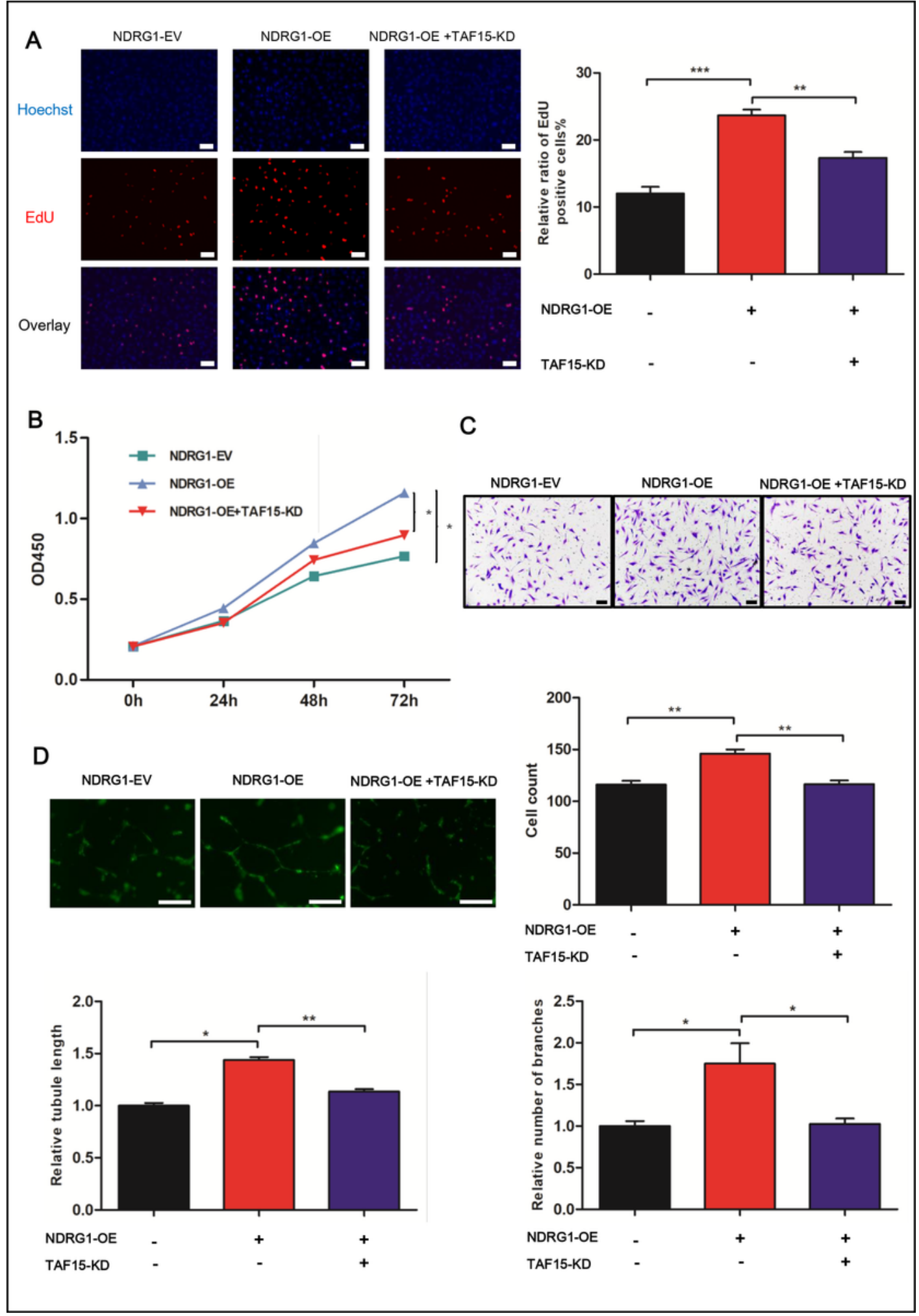




\section{Figure 7}

NDRG1 regulates HPH-related phenotypes of HPAECs through TAF15. (A) HPAECs were transfected with control or NDRG1-overexpressing (OE) constructs, and with TAF15-targeted shRNAs (TAF15-KD), and cell proliferation was measured by EDU assay $24 \mathrm{hrs}$ after transfection. The ratios of red (EdU) to blue (Hoechst) cells were plotted. Scale bar, $100 \mu \mathrm{m}$. (B) Cells were transfected as described in (A), and were subjected to CCK-8 assay. (C, D) HPAECs were transfected as described in (A), and were subjected to measurement for migration by transwell assay (C) and angiogenic response by tube formation (D). ${ }^{*} p<0.05,{ }^{*} p<0.01,{ }^{* \star *} p<0.001$. 
A

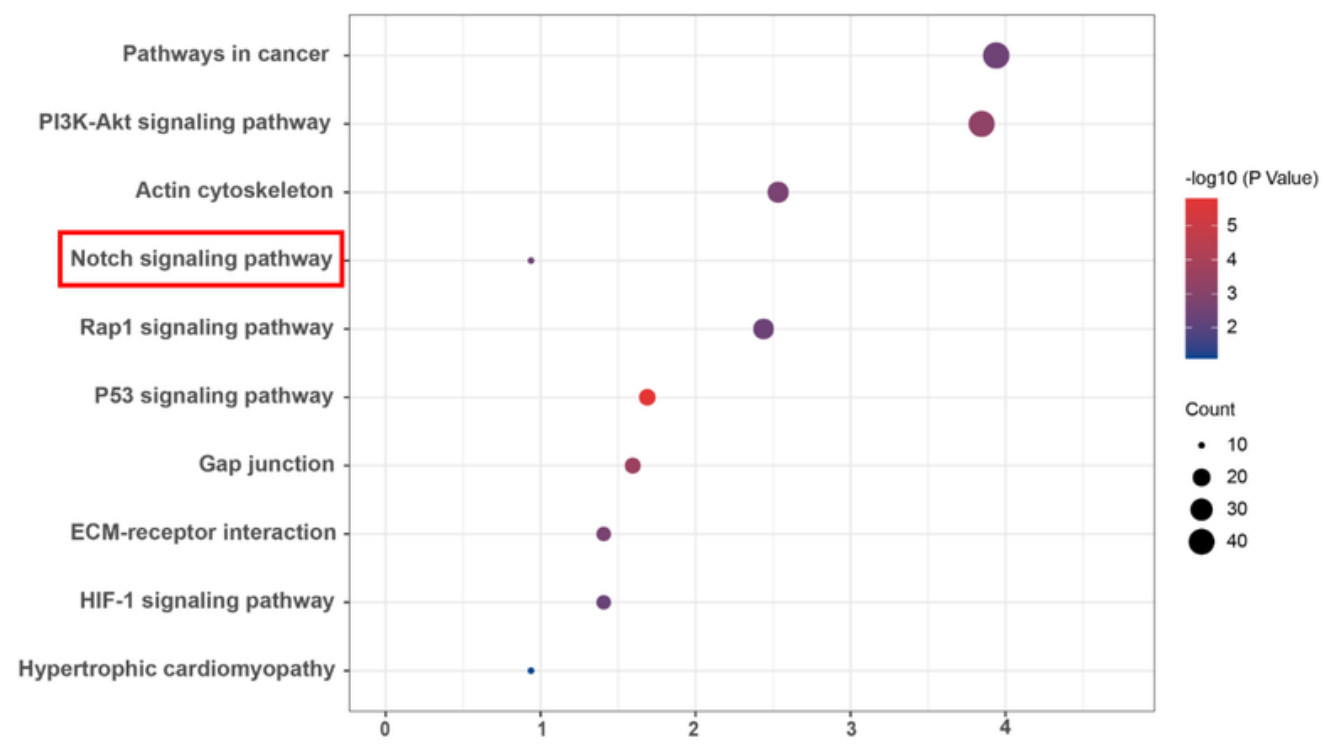

B
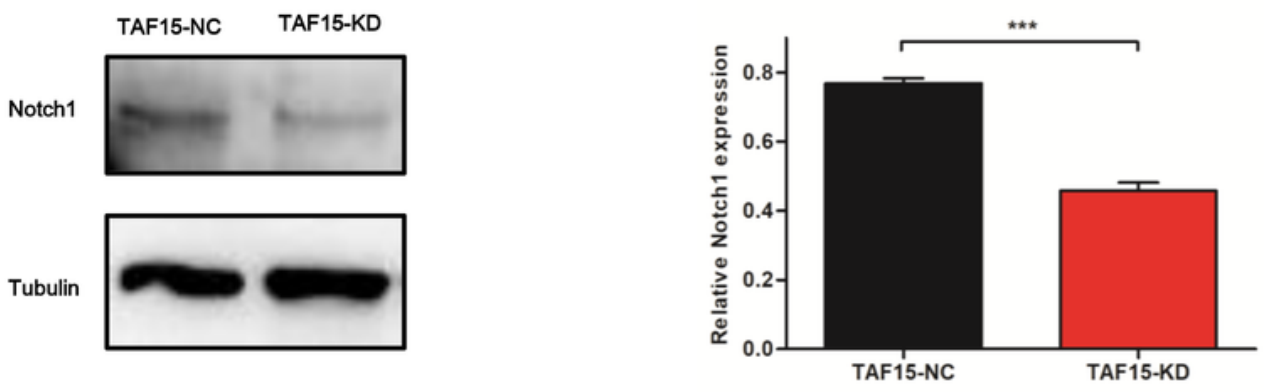

C
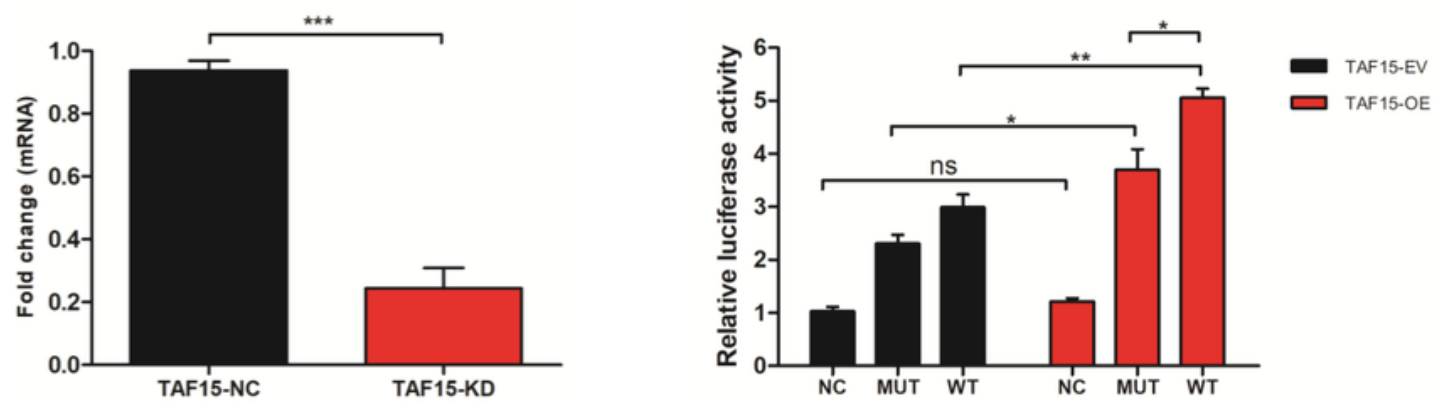

\section{Figure 8}

Notch1 is downstream of the signal pathway of TAF15 in HPAECs. (A) KEGG analysis showed that TAF15 may play a role by regulating PI3K-Akt, p53, HIF-1 and Notch signaling pathway. (B) Western blot analysis of Notch1 in control and TAF15 knockdown HPAECs. Tubulin was blotted as the loading control. (C) qRTPCR assay for Notch1 in control and TAF15 knockdown HPAECs. (D) HEK293T cells were co-transfected with a firefly luciferase reporter construct of human Notch1 promoter, a Renilla luciferase vector and a 

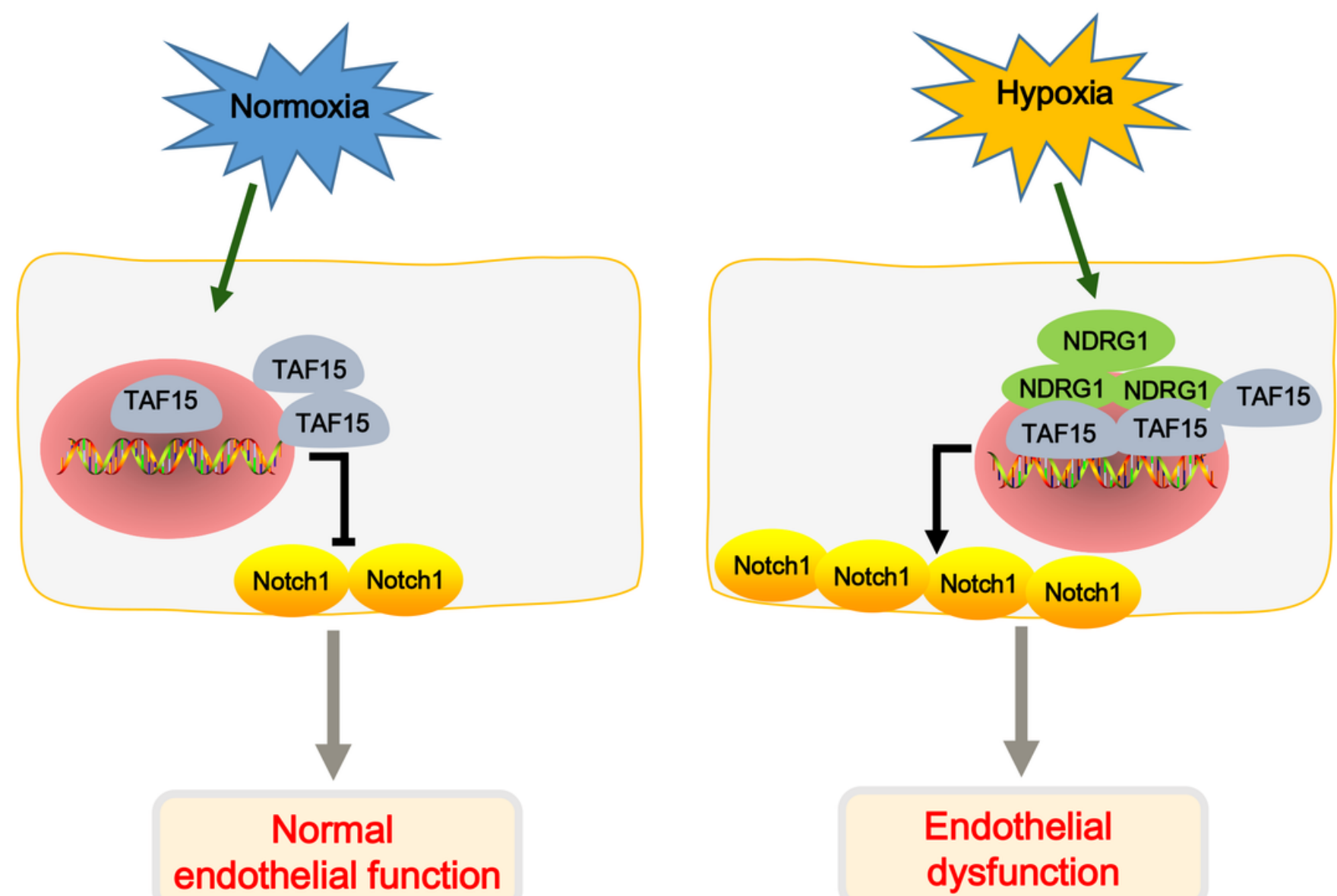

endothelial function

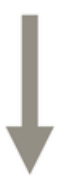

Normal

Pulmonary Circulation
Endothelial dysfunction

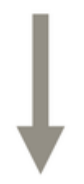

\section{Pulmonary Hypertension}

\section{Figure 9}

A schematic working model. NDRG1 is up-regulated by hypoxia in HPAECs of HPH subjects. NDRG1 functions as a scaffold protein that forms a complex with TAF15, thereby promoting TAF15 nuclear localization and subsequently Notch 1 expression, and contributing to endothelial dysfunction and $\mathrm{HPH}$. 


\section{Supplementary Files}

This is a list of supplementary files associated with this preprint. Click to download.

- Supplementarymaterials.docx 